\title{
The Home-Cage Automated Skilled Reaching Apparatus (HASRA): Individualized Training of Group-Housed Mice in a Single Pellet Reaching Task
}

\author{
Gilles Salameh, DMatthew S. Jeffers, Junzheng Wu, Julian Pitney, and ĐGergely Silasi
}

https://doi.org/10.1523/ENEURO.0242-20.2020

Department of Cellular and Molecular Medicine, Faculty of Medicine, University of Ottawa, Ottawa, Ontario K1H 8M5, Canada

\begin{abstract}
The single pellet reaching task is commonly used in rodents to assess the acquisition of fine motor skill and recovery of function following nervous system injury. Although this task is useful for gauging skilled forelimb use in rodents, the process of training animals is labor intensive and variable across studies and labs. To address these limitations, we developed a single pellet reaching paradigm for training and testing group housed mice within their home cage. Mice enter a training compartment attached to the outside of the cage and retrieve millet seeds presented on a motorized pedestal that can be individually positioned to present seeds to either forelimb. To identify optimal training parameters, we compared task participation and success rates between groups of animals that were presented seeds at two different heights (floor vs mouth height) and at different intervals (fixed-time vs trial-based). The mouth height/fixed interval presentation style was most effective at promoting reaching behavior as all mice reached for seeds within $5 \mathrm{~d}$. Using this paradigm, we assessed stroke-induced deficits in home-cage reaching. Following three weeks of baseline training, reaching success rate was $\sim 40 \%$, with most trials performed during the dark cycle. A forelimb motor cortex stroke significantly decreased interaction with presented seeds within the first $2 \mathrm{~d}$ and impaired reaching success rates for the first $7 \mathrm{~d}$. Our data demonstrate that group-housed mice can be efficiently trained on a single pellet reaching task in the home cage and that this assay is sensitive to stroke induced motor impairments.
\end{abstract}

Key words: behavior; forelimb; motor; mouse; reaching; recovery

\section{Significance Statement}

We developed an automated apparatus and single pellet reach training paradigm for group housed mice within the home cage. This task allows individualized training progression and handedness of presentation for each mouse, minimizes stress induced by experimenter interaction, and enables experiments and data collection on a massive scale, previously impossible because of the demands of one-on-one behavioral testing. We demonstrate an optimized set of task parameters and the utility of this apparatus for assessing lesion-induced motor impairments. Herein, we provide an open-source, scalable, platform for training and assessing skilled reaching in mice with minimal need for experimenter handling that is equivalent to goldstandard, manual single pellet training paradigms.

Received June 4, 2020; accepted September 8, 2020; First published October 2, 2020.

The authors declare no competing financial interests.
Author contributions: M.S.J. and G.Si. designed research; G.Sa. performed research; J.W. and J.P. contributed unpublished reagents/analytic tools; G.Sa., M.S.J., and J.W. analyzed data; G.Sa., M.S.J., and G.Si. wrote the paper. 


\section{Introduction}

The single pellet reaching task is the gold standard for assessing forelimb motor function in rodents. The task requires animals to reach through a slot at the front of a training chamber and retrieve a food pellet that is presented either on a shelf or pedestal (Whishaw and Pellis, 1990; Whishaw, 2000; Farr and Whishaw, 2002; Whishaw et al., 2017). Quantifying this behavior provides several metrics of skilled forelimb use that are commonly used to assess motor learning and gauge impairments after neuronal injury. For example, the rate of task acquisition can be used to assess how various genetic manipulations impact skilled motor learning (Gu et al., 2017; Ueno et al., 2018), while endpoint measures such as reaching success rate are sensitive to acute changes in brain function induced by injury or temporary inactivation (Silasi et al., 2008; Brown and Teskey, 2014; Guo et al., 2015; Clark et al., 2019). Quantification of movement kinematics can also be performed to assess subtle changes in reaching behavior, such as the emergence of compensatory movements after injury (Gharbawie et al., 2005a,b) or alterations in movement subcomponents induced by pharmacological manipulations (Metz et al., 2003).

Although the single pellet reaching paradigm is widely used, there are two main challenges that limit the utility of the task. First, the majority of training requires the experimenter to work with the animals one at a time, limiting the amount of individual training time and number of animals that can be included in experiments. Second, individual differences between experimenters may affect the quality of training and overall reaching success rate that is achieved (Fouad et al., 2013). Because of these challenges, there has been great interest in automating the training process with hopes of increasing throughput and minimizing variability. Several studies have shown that automated training is in fact feasible and has measurable benefits (Fenrich et al., 2015; Ellens et al., 2016; Gu et al., 2017; Torres-Espín et al., 2018; Hubbard et al., 2019). For example, presenting pellets using a motorized delivery arm reduced variability in success rate between and within experiments compared with manually presented pellets (Torres-Espín et al., 2018). Allowing animals to self-initiate trials by activating a sensor within the testing chamber (Wong et al., 2015; Ellens et al., 2016) can be used to further reduce the need for interaction with the experimenter, thus increasing the number of subjects that can be trained simultaneously by running multiple training chambers in

G.Si. was supported by the Natural Sciences and Engineering Research Council of Canada, Canadian Foundation for Innovation, Canadian Institutes of Health Research, and Canadian Partnership for Stroke Recovery.

Acknowledgements: We thank Ralph Nevins for his work on early versions of the task, Wang Xin for his assistance with CAD design, Dr. Matthew Walker for producing the artwork for Figure 1, and Dr. Dale Corbett for his critical review of this manuscript prior to submission.

Correspondence should be addressed to Gergely Silasi at gsilasi@uottawa.ca.

https://doi.org/10.1523/ENEURO.0242-20.2020

Copyright (C) 2020 Salameh et al.

This is an open-access article distributed under the terms of the Creative Commons Attribution 4.0 International license, which permits unrestricted use, distribution and reproduction in any medium provided that the original work is properly attributed. parallel. Although this form of automated training results in reaching success rates that are similar to manual training, one drawback is that animals must still be removed from the home cage by the experimenter, transferred to a testing apparatus, and tested during a specific time of day (typically during the light cycle). In addition to being labor intensive, handling the animals induces a persistent stress response even when habituated (Balcombe et al., 2004), interrupts their rest cycle, and produces periods of social isolation. An alternate approach is to perform training and testing within the home cage (Woodard et al., 2017; Silasi et al., 2018; Bollu et al., 2019; Erskine et al., 2019). In addition to increasing the number of animals that can be tested simultaneously in a single apparatus, home cage testing facilitates self-initiated task participation during the natural active (dark) cycle of the animals without having to reverse the light cycle of the animal facility.

Based on these advantages, we developed a homecage automated skilled reaching apparatus (HASRA) that can be used to train group-housed mice to a reaching success rate similar to manual training using the singleseed variant of the task (Xu et al., 2009). This minimizes stress to the animals by limiting the presence of experimenters during testing. We systematically modulated two key training parameters, pellet presentation height and timing of trial presentation, to determine optimal training parameters. Using this system, we then quantified reaching impairments in a group of mice that received a photothrombotic motor cortex stroke.

\section{Materials and Methods}

\section{HASRA}

Rodents display strong lateralization of limb use, preferring to reach with either their left or right hand (Whishaw, 1992). Furthermore, training in the single pellet task typically involves initially allowing retrieval of pellets in a proximal position with either the tongue or hand and progressively shaping the rodent to begin using only the hand to retrieve pellets from a more distal position (Whishaw et al., 2008). In order to accommodate these strategies among group-housed mice, it was crucial that our device be able to deliver the pellet in positions that were individualized to each animal's hand preference and training stage.

Two stepper-motors (Pololu, 1205) controlled the proximal-distal and medial-lateral positioning of a seed presentation arm and hopper apparatus inspired from a previously published design (Torres-Espín et al., 2018). The presentation arm's movement in both axes could be adjusted within $\mathrm{a} \sim 1-\mathrm{cm}$ range relative to the front slot $(3.5 \mathrm{~cm}$ tall $\times 1.0 \mathrm{~cm}$ wide; Fig. $1 A)$ that the mice reached through. A servo motor (Hitec, HS-82MG) mounted to the seed hopper controlled the speed and height of the seed presentation arm relative to the entry tube's floor level. Upon activation, the seed delivery arm was initially immersed in the seed hopper and traveled up to its set height. $A \sim 1-m m$-deep well in the tip of this arm ensured presentation of a single seed. All motors were controlled 


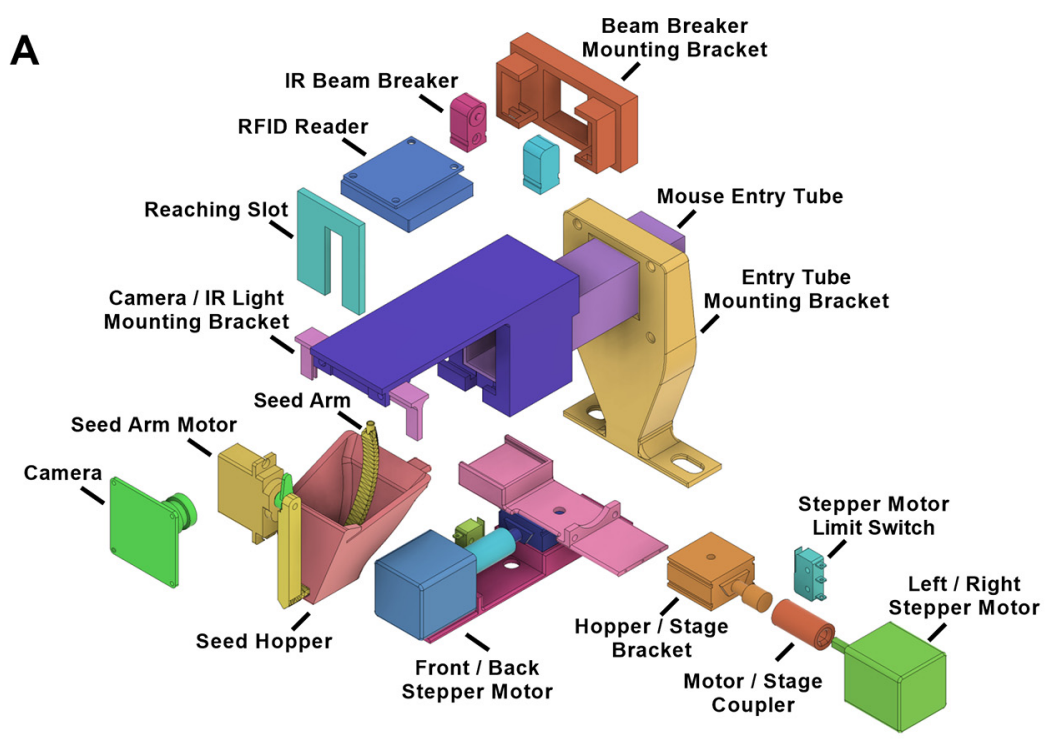

B

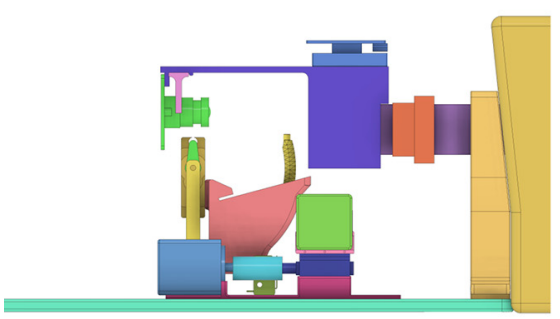

D Lick

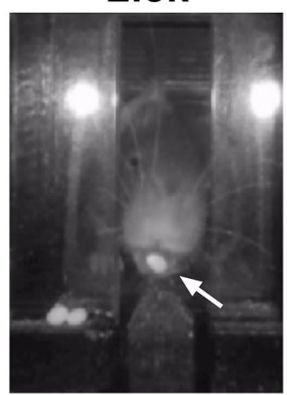

Miss

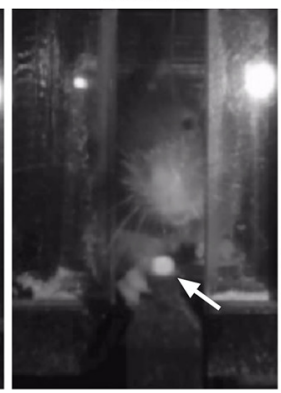

C

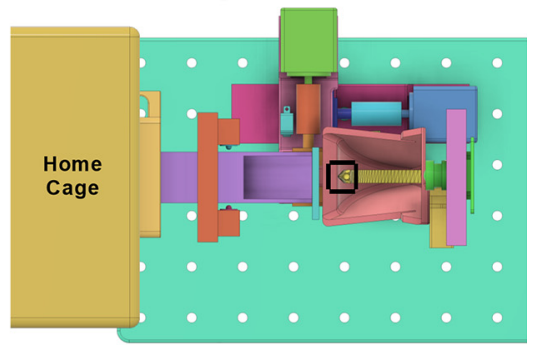

Knockdown Success
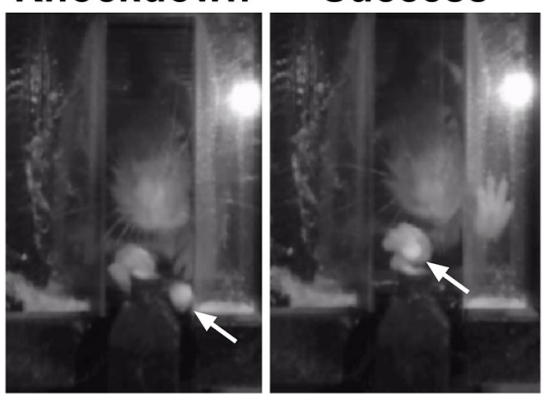

Figure 1. HASRA. A, Exploded view of apparatus, showing each part of the assembly. $\boldsymbol{B}$, Side view of apparatus, showing relative positions of reaching compartment, seed arm, camera, and RFID tag reader for identifying mice. $\boldsymbol{C}$, Top-down view of front wall, stage motors and range of motion in which a seed could be presented (within black bounding box). $\boldsymbol{D}$, Representative images taken from wallfacing camera showing a mouse performing each type of event that was classified. Miss, knock-down, and success represented types of attempts to retrieve the pellet using the hand. This perspective was used for scoring data on mouse reaching performance. Location of seed indicated with white arrow in each image.

by an Arduino Nano (Arduino, 7630049200173). Most parts, excluding electronics, were 3D printed in the laboratory using a fused deposition modeling printer (FlashForge 3D printer, Creator Pro). The front wall reaching slot was cut from acrylic using an Epilog laser cutter. Up-to-date part listings and instructions to build the device (including Python/Arduino code, 3D printing STL files, and wiring diagrams) can be found at https://github. com/SilasiLab/HASRA. An archived version of these files at the time of publishing can be found attached to this article (Extended Data 1).

\section{Radio frequency identification (RFID) tag insertion and HASRA activation}

All mice were subcutaneously implanted with a unique RFID tag (Sparkfun, SEN-09416) enabling individual identification. Animals were briefly $(1-2 \mathrm{~min})$ anesthetized using $4 \%$ isoflurane in room air and injected between the scapula with a sterile RFID tag. Upon regaining mobility, mice were introduced to the new home cage and immediately had access to the HASRA.

A $10-\mathrm{cm}$ acrylic tube $(2.54 \times 2.54 \mathrm{~cm})$ sized to fit one mouse at a time was attached to the home cage, leading 


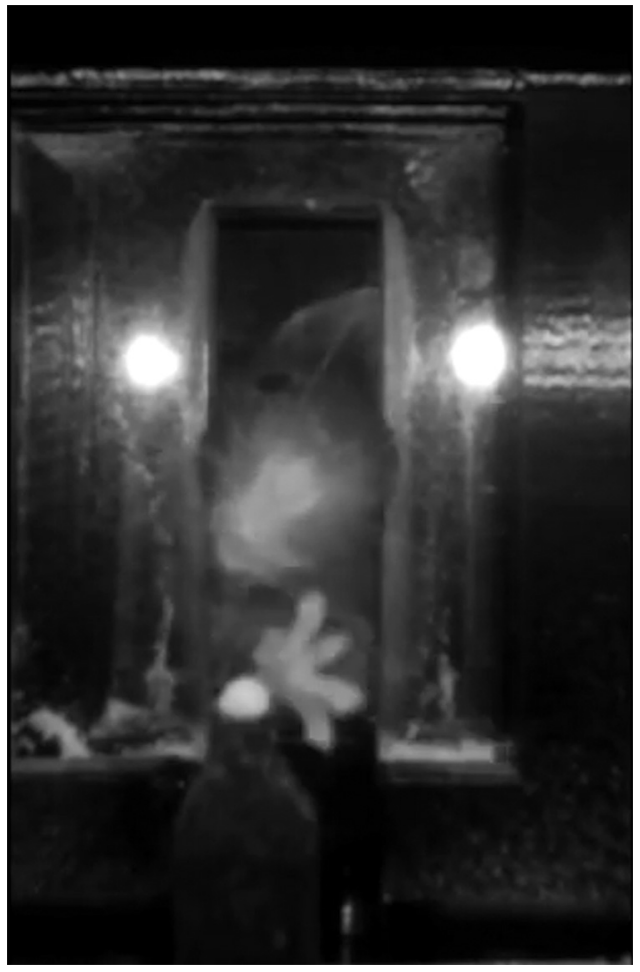

Movie 1. Example video showing a mouse engaging with the seed delivery arm to retrieve millet seeds. [View online]

to a front wall with a slot through which animals could reach for millet seeds (Chen et al., 2014). The entry of a mouse into the tube initiated a trial by interrupting an infrared beam projecting across the width of the tube (Adafruit, 2167). Our custom Python script sampled the RFID reader (Sparkfun, SEN-11827) mounted on top of the tube (Fig. $1 B$ ) to identify the animal and triggered the motorized hopper and seed presentation arm to move to a user defined position and present a millet seed (Fig. 1C). Each presentation was recorded using a video camera directly facing the reaching slot. When the mouse exited the tube (detected by closed IR breaker), the seed hopper and presentation arm returned to a home position and video recording was discontinued.

\section{Video recording and seed detection}

Detection of the mouse by the RFID tag reader activated a video camera (ELP, USBFHD08S-LC1100) equipped with an adjustable focus lens, which recorded at $60 \mathrm{fps}$ until the mouse exited the tube. Two infrared light sources (HonYan, 191094177157) flanked the camera lens to enable recording during the dark cycle. A Python script automatically uploaded videos (where a minimum of one seed was presented) to a Google Drive folder corresponding to the mouse's RFID tag (Movie 1). This enabled the experimenter to remotely monitor mouse activity and quantify videos without overwhelming local storage capacity. Videos where a mouse exited the tube before at least one seed could be presented were automatically deleted.

A neural network was used to continually scan the video feed for the presence/absence of a seed. This neural network was a variation of the MobileNetV2 architecture (Sandler et al., 2018). To train the network, a dataset of $N=5482$ video frames was collected from HASRA. These frames were manually labeled as either containing a seed or not containing a seed. In an effort to increase the variability of this small training dataset (and therefore increase the ability of the trained network to generalize to novel examples), these labeled frames were subjected to standard supervised machine learning dataset augmentation techniques including rotations, scaling and brightness adjustments. The dataset was then partitioned into validation and training partitions containing $10 \%$ and $90 \%$ of the total frames, respectively. The network was then trained on the training partition for 50 epochs with a batch size of 32. The trained network reached an accuracy of $99.08 \%$ on the validation partition. The network was then deployed to the HASRA where it was used to detect the presence/absence of seeds in real time. This real-time detection was used to trigger the presentation of a new seed whenever no seed was detected for $\geq 4 \mathrm{~s}$.

Automated detection of successful seed presentation also enabled a method to benchmark the mechanical success rate of presentation for a given arm (successful presentation rate was checked daily, and maintenance was performed when successful presentation dropped below 90\%). Maintenance activities consisted of filling the hopper with seeds, removing crumbs from the $\sim 1$-mm-deep well at the tip of the motorized arm and bottom of the hopper using a brush and completely replacing the arm with a new one if presentation rate could not be otherwise improved. Additionally, if mice had caused any damage to the front wall of the reaching slot by chewing then this would be replaced with a new front wall. These maintenance activities required the HASRA to be taken offline for no more than a few minutes. No other mechanical issues were observed during these experiments, although we have observed that it is possible for the motors and mechanical stages to eventually fail after months of continual use, at which point they can be easily replaced.

\section{Validation experiment design}

A total of 27 Thy1-ChR2-YFP mice (The Jackson Laboratory, stock \#007612), aged between 44 and $80 \mathrm{~d}$ old, were housed in groups of three to five on a 12/12 h light/dark cycle. Standard mouse chow was restricted to $1 \mathrm{~g} / \mathrm{mouse} / \mathrm{d}$, meaning that for a cage of five mice, we provided $5 \mathrm{~g}$ of chow per day. Mice were able to supplement their diet with millet seeds ad libitum by successfully using the HASRA. Although we were not able to track the exact amount of chow that each mouse consumed, we broke the pellets into small pieces and spread them throughout each cage to allow all mice a chance to forage and obtain it. Water was accessible ad libitum. Animals were weighed weekly to monitor their body weight. All procedures were performed in accordance with our Animal Care Committee and complied with Canadian Council on Animal Care (CCAC) guidelines.

In experiment 1, we determined the optimal seed arm height (floor height vs mouth height) and presentation 
style (continuous 5-s cycling vs stationary presentation until seed no longer detected) to maximize task engagement, progression from licking to reaching behavior, and reaching success rate within the HASRA. This was conducted over a two-week period with four groups: (1) floor height with continuous cycle (low cycle, $n=5$ female mice); (2) floor height with stationary presentation (low single, $n=5$ female mice); (3) mouth height $(0.8 \mathrm{~cm}$ above the floor) with continuous cycle (high cycle, $n=5$ female mice); and (4) mouth height with stationary presentation (high single, $n=5$ female mice).

In experiment 2, a second group of mice using the factorial combination with the greatest success in experiment 1 (high cycle) was trained over a three-week period $(n=7$ male mice). This replicated and extended the training curve obtained in experiment 1 . At the conclusion of training, mice were randomly assigned to receive either a photothrombotic stroke $(n=4)$ or a sham surgery $(n=3)$. Mice were allowed continual access to the HASRA for $7 \mathrm{~d}$ following stroke, with their performance quantified to assess the sensitivity of this task to stroke-induced impairments of skilled reaching and grasping.

\section{Daily qualitative scoring}

Daily qualitative scoring was used to describe the animals' performance and engagement with the HASRA. Approximately five videos per mouse were evaluated each day and attributed a score from 1 to 4 . A score of 1 was credited to a mouse not showing any licking or reaching behavior. If an animal was seen only licking the seed displayed in front of the wall at least once, it received a score of 2. A score of three represented a combination of licking and reaching behavior. Finally, a mouse that solely reached for the seed obtained a score of 4 . This score was used to assess the animals' behavior and inform the experimenter on each animal's training stage for the adjustment of the arm's position each day.

\section{Training stages}

Stage 1

Upon initial introduction to the HASRA, the presentation arm was set to deliver seeds at the closest distance possible $(0.4 \mathrm{~cm}$ from front wall) and in the center of the front wall slot to encourage licking of the seed. This was intended to habituate animals to the HASRA, and millet seeds presented outside the front wall. An animal moved to stage 2 only if it was observed using its tongue or hand to successfully retrieve a seed.

\section{Stage 2}

Once seed retrieval was observed, the presentation arm was progressively moved further away from the front wall $(\sim 0.4 \mathrm{~cm} / \mathrm{d}$ up to a maximum of $1.5 \mathrm{~cm})$ until the animal could no longer retrieve the seed by licking. This stage was intended to eliminate licking behavior and train animals to use their hand to reach and grasp the seed. An animal completed this stage when it frequently used its hand for seed retrieval.

\section{Stage 3}

Once an animal retrieved the seed by reaching and grasping with its hand, seeds were presented with a left or right offset relative to the edge of the slot opposing the preferred hand $(\sim 0.5 \mathrm{~cm}$ off-center) as is done during manual training (Farr and Whishaw, 2002). During this final stage animals developed success at skilled reaching by repetitively performing the task using their preferred hand.

\section{Reaching success rate}

The reaching success of each animal was quantified by scoring 20 events per mouse at each desired time point (weekly in experiment 1, every second day in experiment 2). Each reaching event was classified into one of four categories: lick, miss, knock-down, success (Fig. 1D). A "lick" was any pellet retrieval that was completed using the tongue and not the hand. An "attempt" was defined as any action where the mouse moved its hand beyond the front wall slot. Several subcategories of attempt were also defined (miss, knockdown, success). A "miss" was defined as an attempt where the movement of the hand did not disrupt the position of the seed. A "knock-down" was defined as an attempt that displaced the seed from the arm but did not successfully retrieve it. A "success" was an attempt in which the seed was grasped and retrieved using the hand to the animal's mouth. Success rate was calculated as: (number of success/number of attempts) $\times 100$.

\section{Photothrombotic stroke induction}

Mice were anaesthetized using $4-5 \%$ isoflurane and secured in a stereotaxic frame using ear bars. An incision was made along the midline of the scalp to expose the skull. A total of $100 \mathrm{mg} / \mathrm{kg}$ of rose bengal (SigmaAldrich, 330000) diluted in PBS was injected intraperitoneally and allowed to circulate for $2 \mathrm{~min}$. A green laser light (532 nm, $1.5 \mathrm{~mm}$ in diameter, $20 \mathrm{~mW}$ power) was positioned $1 \mathrm{~cm}$ anterior, and $\pm 1.5 \mathrm{~cm}$ lateral to bregma (in the hemisphere contralateral to the preferred hand). This positioning corresponded to the forelimb motor region in the mouse (Tennant et al., 2011) and was intended to specifically impair reaching and grasping ability. The laser was positioned $5 \mathrm{~cm}$ above the surface of the skull and activated for $13 \mathrm{~min}$ (Balbi et al., 2017). Sham mice received the laser illumination first, followed by the rose bengal injection after the laser was turned off. This order of events exposes the sham mice to exactly the same procedures as the stroke mice but does not result in any lesion. Afterwards, the scalp was sutured, bupivacaine was applied on the incision site as a topical analgesic, and mice regained mobility before being returned to their home cage. At 6 - and $24-\mathrm{h}$ following surgery, mice had bupivacaine reapplied to their incision site. Following the stroke surgery, animals were reintroduced to the HASRA for one week. The position of the seed arm was 
left unchanged from where it was before stroke. The stroke surgery did not result in any mortality.

\section{Assessment of lesion size and location}

Following the conclusion of the experiment, mice were deeply anesthetized with sodium pentobarbital, decapitated, and their heads placed in $10 \%$ neutral buffered formalin at $4^{\circ} \mathrm{C}$ for fourmonths. Brains were then removed and cryoprotected in $30 \%$ sucrose in PBS until saturated. Brains were then frozen at $-80^{\circ} \mathrm{C}$ and every second coronal section $(50 \mu \mathrm{m} /$ section $)$ from the olfactory bulb to the posterior portion of the hippocampus was mounted on gelatin-coated slides and stained with cresyl violet. Slides were scanned using a flatbed slide scanner (Canon 900F MKII) at a resolution of $1200 \mathrm{dpi}$. The area of damaged tissue was delineated on each section using ImageJ, with infarct volume calculated as: $\Sigma$ (area of damage on each section) $\times$ tissue volume between each section $(100 \mu \mathrm{m})$.

\section{Statistical analysis}

All statistical analysis was performed using SPSS Statistics (v26, IBM Corp). In experiment 1, separate Kruskal-Wallis tests were used to assess task engagement on day 1 of training in each of the individual groups (low cycle, low single, high cycle, high single), and also collapsed across the arm height (low vs high) and presentation style (cycle vs single) variables. Total time spent in the task each day, daily qualitative score, and mean success rate were analyzed using repeated-measures ANOVA, with training day as a within-subjects factor and both arm height and presentation style as between-subjects factors. The Greenhouse-Geisser correction was applied to all repeated-measures analyses. Post hoc analysis was performed using Sidak-corrected $t$ tests. Family-wise Type I error was controlled within each outcome measure to $\alpha=0.05$ as the significance cutoff. Proportion of each group that showed reaching at least once by a given day was analyzed using Log-rank (Mantel-Cox) $\chi^{2}$ survival tests. In experiment 2, total time spent in the task each day, daily qualitative score, and mean success rate were analyzed using the same repeated-measures ANOVA methodology as used for experiment 1.

\section{Results}

\section{HASRA parameter optimization}

\section{Task engagement}

To assess the ability of the HASRA to initially engage animals to use the task, the daily qualitative score on day 1 was used as a metric of task engagement. A significant interaction between arm height and presentation style $\left(H_{(3)}=15.813, p=0.001\right.$; Fig. $2 A$, left $)$ indicated that the low cycle and low single groups had equivalently low levels of engagement $(0 \%$ and $20 \%$, respectively), while the high cycle and high single groups had significantly greater levels of early engagement $(100 \%$ in both groups, with high single having $60 \%$ of mice both licking and reaching on day 1). Overall, arm height had a significant impact on task engagement $\left(H_{(1)}=14.646, p<0.001\right.$; Fig. $2 A$, center), with $10 \%$ of animals with the low (floor height) arm, and $100 \%$ with the high $(0.8 \mathrm{~cm}$ above floor) arm engaged in the task on day 1. Conversely, seed presentation style did not significantly impact day 1 engagement $\left(H_{(1)}=\right.$ $1.058, p=0.304$; Fig. $2 A$, right) with $50 \%$ engagement with a cycling style, and $60 \%$ engagement with a single presentation style.

The ability to not only engage animals early in training but also to sustain their interest in using the HASRA long-term (across weeks) was deemed essential. This was quantified using the total time each group spent using the task across days. No arm height by presentation style interaction was observed $\left(F_{(5.48,87.61)}=1.772, p=0.121 ;\right.$ Fig. $\left.2 B\right)$, but significant main effects of both arm height $\left(F_{(5.48,87.61)}=3.529\right.$, $p=0.005)$ and presentation style $\left(F_{(5.48,87.61)}=2.454\right.$, $p=0.035$ ) were observed. Post hoc analysis of these effects indicated that the high arm height resulted in greater time-on-task on days 1,3 , and $9(p<0.05)$, whereas cycling presentation style resulted in greater time-on-task on days 6 and 9-14 $(p<0.05)$.

\section{Adoption of reaching strategy}

We sought to identify the HASRA parameters that would result in the greatest proportion of mice adopting reaching behavior (daily qualitative score $\geq 3$ ) in the fewest number of days possible. There was a significant interaction among the time, arm height, and presentation style factors $\left(F_{(4.13,66.07)}=2.643, p=0.040\right)$, with the mean qualitative score of the high arm across time being significantly greater than that of the low arm $\left(F_{(1,16)}=17.899\right.$, $p=0.001$; Fig. $2 C$ ). In comparison, the single and cyclical presentation styles weren't statistically different overall $\left(F_{(1,16)}=0.479, p=0.499\right)$.

The day by which each group first displayed reaching behavior (daily qualitative score $\geq 3$ ) was significantly different between the four groups $\left(\chi_{(3)}^{2}=21.38, p<0.0001\right.$; Fig. 2D). The high presentation showed a significantly greater adoption of reaching behavior (90\% of group by day 5) compared with the low presentation (50\% of group by day $\left.12, \chi^{2}(1)=7.752, p=0.009\right)$. However, the single ( $70 \%$ by day 8$)$ and cyclical $(60 \%$ by day 5$)$ presentations styles did not significantly differ $\left(\chi^{2}{ }_{(1)}=0.298, p=0.585\right)$ and achieved similar proportions of animals displaying reaching behavior (Fig. 2D). Overall, the high single group achieved both the greatest and most rapid adoption of reaching behavior, with $100 \%$ of the group having shown reaching behavior by day 4 .

\section{Reaching success rate}

Success rate was a quantitative assessment of the percentage of successful reaches among the 20 events scored for each mouse every $7 \mathrm{~d}$. A significant time by arm height by presentation style interaction $\left(F_{(1,16)}=\right.$ $7.750, p=0.013$ ) indicated that the high cycle group was the only one to significantly improve its reaching success rate by day 14 (Fig. 2E). Beneficial main effects of high arm height $\left(F_{(1,16)}=4.766, p=0.044\right)$ and cyclical presentation style $\left(F_{(1,16)}=10.468, p=0.005\right)$ were also observed. Overall, the high single group had displayed the 

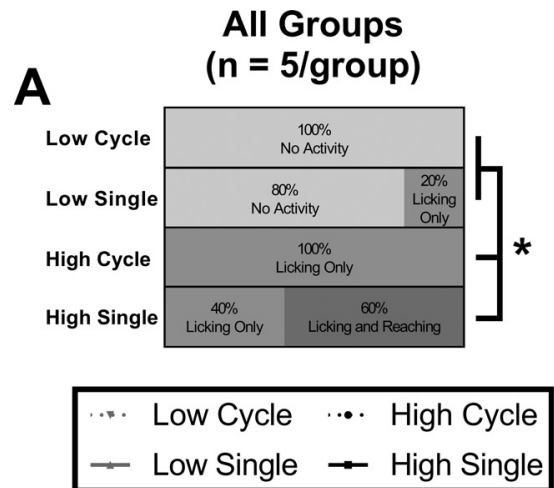

B

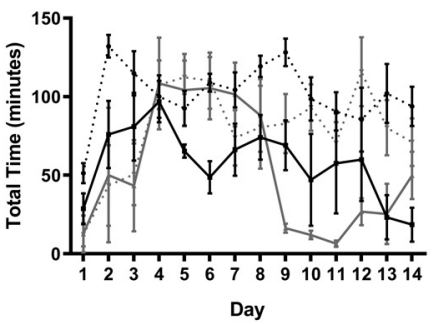

C

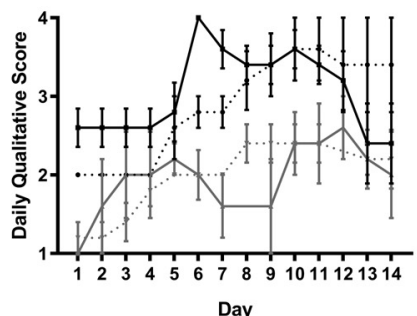

D

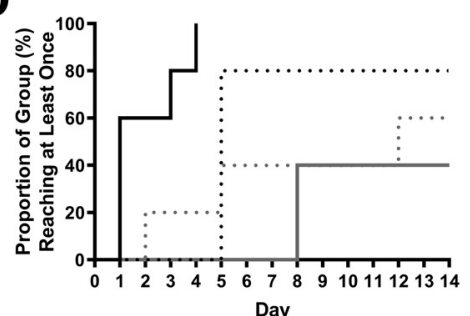

E

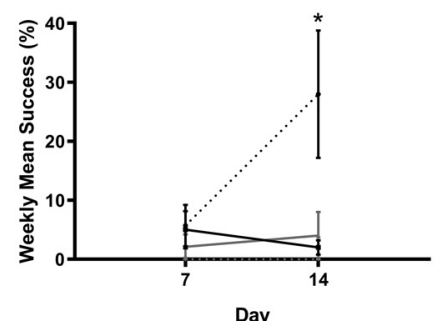

Low vs High

( $n=10 /$ group)
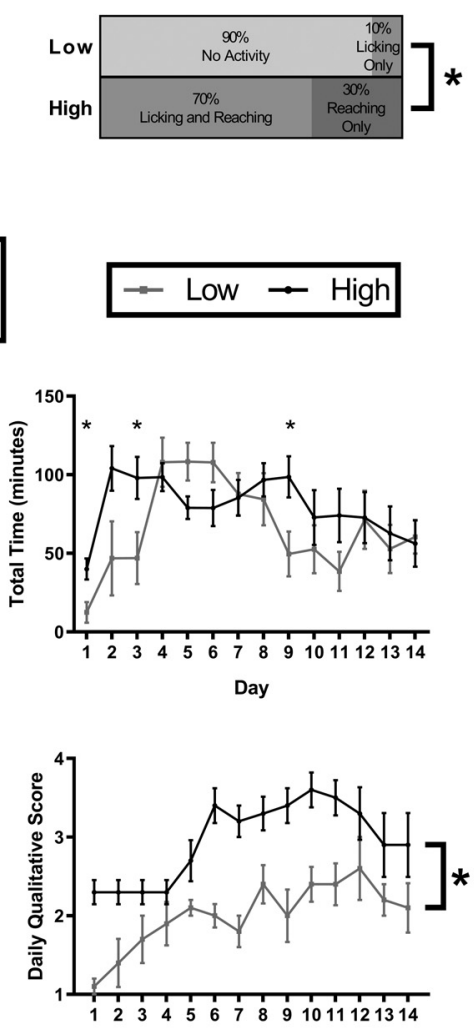

Day

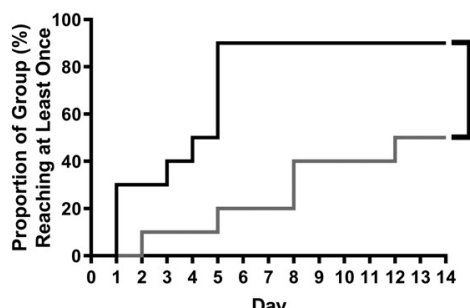

Day

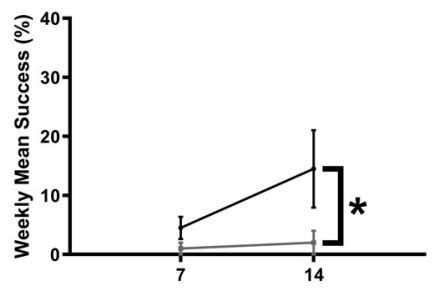

Day

\section{Cycle vs Single \\ ( $n=10 /$ group)}

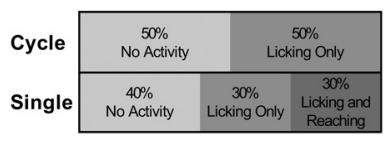

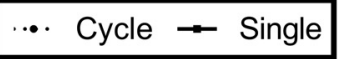

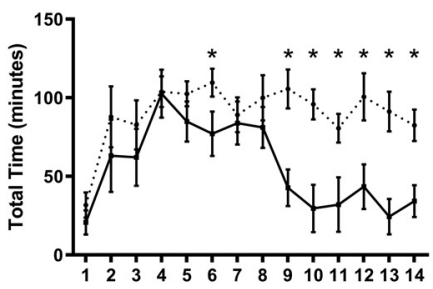

Day

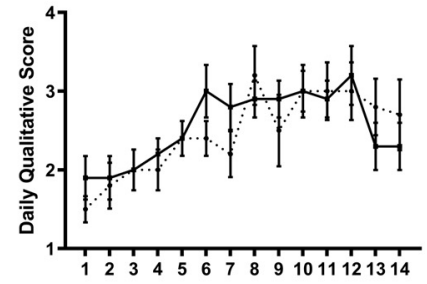

Day

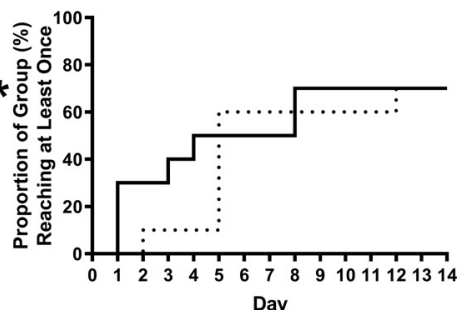

Day

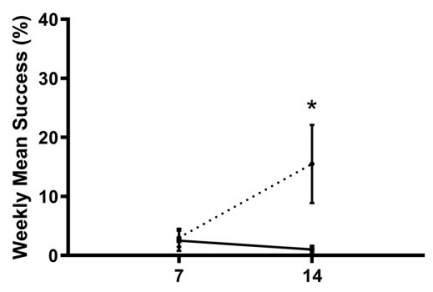

Day

Figure 2. HASRA parameter optimization. A, Proportion of each group showing varying behaviors on day 1 of exposure to the task. $\boldsymbol{B}$, Mean time per day (minutes) per mouse in which the pellet arm was delivering pellets for each group. This measure of time was used to avoid counting time in which the mouse rapidly entered and exited the HASRA without waiting to actually be delivered at least one pellet. Time in which the animal remained focused on the task long enough to be delivered a pellet more accurately represents, "task engagement." $\boldsymbol{C}$, Mean daily qualitative score for each group based on scoring five videos per animal per day. $\boldsymbol{D}$, Survival curve of percentage of mice that displayed a qualitative score of at least 3 (reaching or licking) by day of training. $\boldsymbol{E}$, Percentage of successful reaching events based on scoring of 20 events per mouse at each time point. All data are represented as mean $\pm \mathrm{SEM}$; represents a post hoc statistical effect of $p<0.05$. 
Table 1: Total reaching event counts by phase of light cycle ${ }^{a}$

\begin{tabular}{lcrrr}
\hline Determinant & Total attempts & Miss & $\begin{array}{c}\text { Reaching event type } \\
\text { Knock-down }\end{array}$ & Success \\
\hline Light phase & 715 & $* 100(14.0 \%)$ & $* 335(46.8 \%)$ & $280(39.2 \%)$ \\
Dark phase & 3285 & $* 362(11.0 \%)$ & $* 1705(51.9 \%)$ & $1218(37.1 \%)$ \\
Total & 4000 & $462(11.5 \%)$ & $2040(51.0 \%)$ & $1498(37.5 \%)$
\end{tabular}

a Total count of each type of reaching event on day 22 of training, split by phase of the light cycle. An attempt was any event where the hand passed through the front slot, resulting in either a miss, knock-down, or successful retrieval of the seed. The number in parentheses shows the percentage of that type of event within a given row. $\chi^{2}$ tests were used to assess differences in the relative distribution of event types across the light cycle (light phase vs dark phase). Cells prefixed by a $*$ had significant differences in their relative distribution between light phases. Overall, $82.1 \%$ of reaching events occurred during the dark phase.

greatest task engagement on day 1 and the most rapid adoption of reaching behavior. However, the significantly greater reaching success rate of the high cycle group at day 14 was deemed to be the most important element of task learning. Therefore, the high cycle parameters were selected for replication and testing for sensitivity to lesion-induced impairments in experiment 2.

\section{Distribution of events by circadian phase}

We were also interested in when mice performed their reaching, and whether animals were relatively more successful at reaching during one phase of the light/dark cycle. In experiment 2, at day 22 (day before stroke), we manually scored all events from all animals ( $N=5159$ events). Mice were well trained by day 22; obtaining the seed by licking in $22 \%$ of the events (1159) and attempting to obtain the seed using their hand in $78 \%$ of events (4000). Of this subset of reaching events, $11.5 \%$ (462) were misses, 51.0\% (2040) were knock-downs, and 37.5\% (1498) were successful retrievals (Table 1 ).

Mice displayed continuous activity in the HASRA during the dark phase, whereas during the light phase mice displayed a period of complete inactivity from $\sim 12$ to 3 P.M. (Fig. 3A). Overall, mice performed significantly more of all types of events in the dark phase than in the light phase (lick, $t_{(3)}=-4.808, p=0.012 ;$ miss, $t_{(6)}=-3.787$, $p=0.009$; knock-down, $t_{(6)}=-5.681, p=0.001$; success, $t_{(5)}=-4.900, p=0.004$; Fig. $3 B$ ). In both phases, the relative percentage of successful attempts did not significantly differ; however, misses were significantly more frequent during the light phase, whereas knock-downs happened significantly more frequently during the dark phase $\left(\chi_{(3)}^{2}=8.089, p=0.018\right.$; Table 1).

\section{HASRA sensitivity to lesion-induced impairments}

In experiment 2 , mice received $22 \mathrm{~d}$ of exposure to the HASRA before receiving a photothrombotic stroke (or sham surgery on day 23) and seven additional days of testing. Mice had their qualitative score assessed daily throughout this period, and their success rate (based on 20 events) scored every second day (Fig. 4A). No significant differences in time-on-task were observed between the sham and stroke groups, neither before, nor after surgery $\left(F_{(2.05,10.24)}=1.184, p=0.346\right.$; Fig. $\left.4 B\right)$. Despite suffering a stroke, mice were still able to engage with the HASRA. Daily qualitative scoring indicated that the stroke group performed significantly worse than the shams on days 6 and 7 of training, but then caught up by day 23 when stroke occurred. Stroke resulted in a drastic reduction in qualitative performance on the day-of, and dayafter stroke, that then rapidly recovered $\left(F_{(3.13,12.51)}=\right.$
A

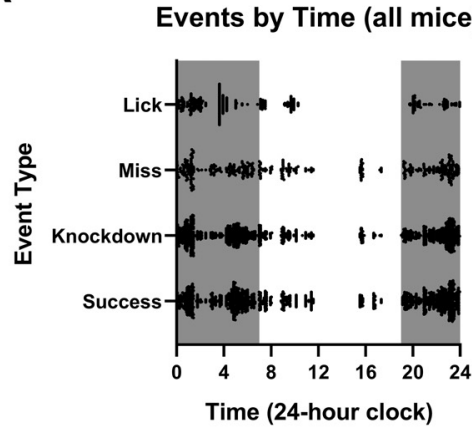

B

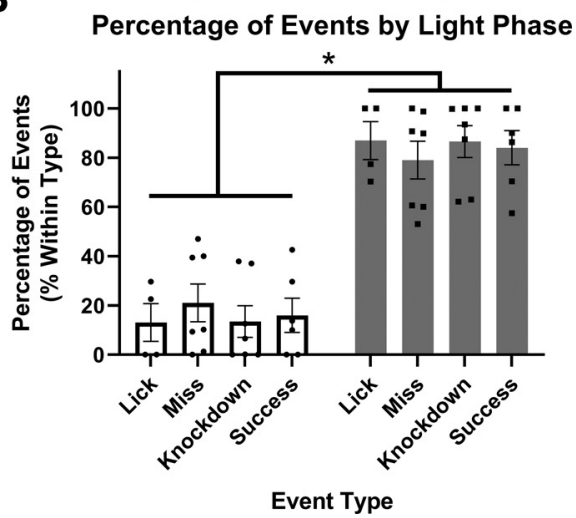

Figure 3. Distribution of events by circadian phase. A, Plot of all events for all mice on day 22 (day before stroke) of experiment 2. Each event of a given type is represented by a single black dot. The white portion of each graph represents the light phase of the light cycle (7 A.M. to 7 P.M.), whereas data in the gray portion of the graph represent data from the dark phase (7 P.M. to 7 A.M.). B, Mean percentage of each event type by portion of the light phase. Mean for individual mice are represented by single dots. $N=5159$ events, $n=7$ mice. All data are represented as mean $\pm \mathrm{SEM}$; represents a post hoc statistical effect of $p<0.05$. 
A

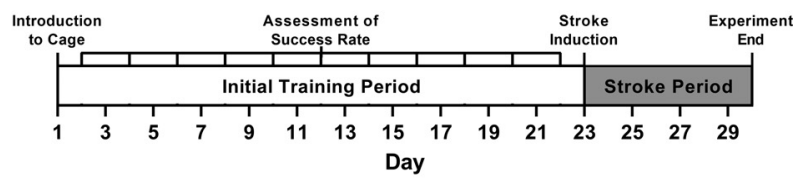

B

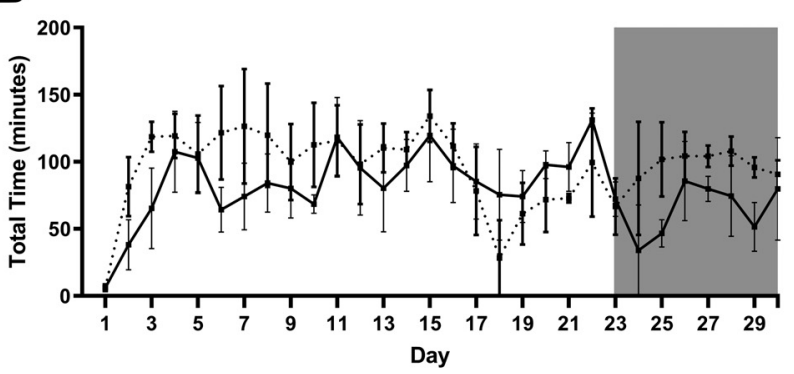

C

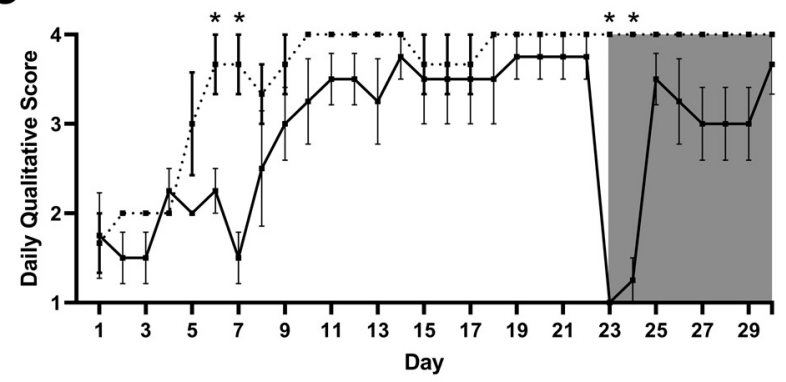

D

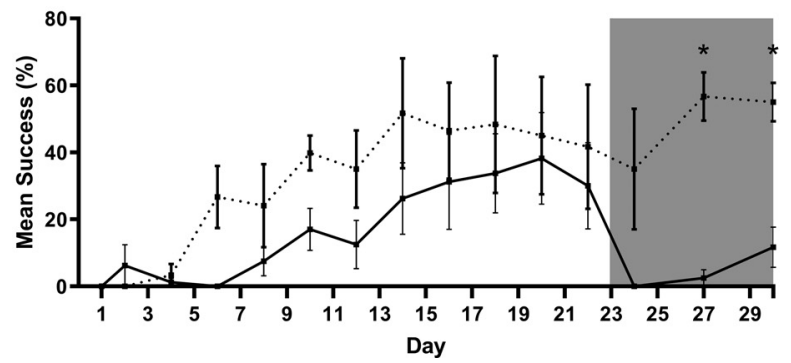

Figure 4. HASRA sensitivity to lesion-induced impairments. $\boldsymbol{A}$, Timeline of experiment 2. B, Mean time per day (minutes) per mouse with the pellet arm activated for each group. $\boldsymbol{C}$, Mean daily qualitative score for each group based on scoring five videos per animal per day. $\boldsymbol{D}$, Percentage of successful reaching events based on scoring of 20 events per mouse at each time point. The white portion of each graph represents time points before stroke, whereas data in the gray portion of the graph represent poststroke time points. All data are represented as mean \pm SEM. $N=7$ for all panels; * represents a post hoc statistical effect of $p<0.05$.

5.951, $p=0.009$; Fig. 4C). On these days, despite spending significant amounts of time in the HASRA, stroke mice were unable to recover seeds, even by licking. Finally, success rates were not significantly different between groups before stroke; however, stroke animals displayed a significant decrease in performance at all points following stroke, and were significantly impaired relative to shams at days 27 and $29\left(F_{(13,52)}=2.721, p=0.005\right.$; Fig. $\left.4 D\right)$.

\section{Verification of animal health and lesion induction}

Throughout experiment 2, body weights were monitored to ensure that no significant reduction in body weight occurred because of food restriction (Fig. 5A). All animals maintained healthy body weights with no adjustment in food ration required. Photothrombotic stroke resulted in a mean lesion volume of $3.59 \pm 0.54$ $\mathrm{mm}^{3}$ (Fig. 5B) with a maximum extent from $+2.4 \mathrm{~mm}$ anterior to $-0.8 \mathrm{~mm}$ posterior to bregma (Fig. $5 \mathrm{C}$ ). These lesions were centered in the region comprising the forelimb motor region of the mouse (Fig. 5D; Tennant et al., 2011).

\section{Discussion}

We developed an automated system for delivering single pellet reach training in a home-cage housing environment and validated the efficacy of this device in two experiments. In experiment 1 , we demonstrated that delivering seeds $0.8 \mathrm{~cm}$ above the tube floor (at approximately mouth level) using a cyclical pattern (every $5 \mathrm{~s}$ ) resulted in the greatest reaching success rate after two weeks of training. This target height is similar to that used in the classic single-pellet reaching task (Farr and Whishaw, 2002) and resulted in $100 \%$ of mice engaging with the task and obtaining seeds on the first day of exposure to the HASRA. This is notable, as it demonstrates that the HASRA rapidly engages mice in the task even without prior habituation to the millet seeds or caging environment. $80 \%$ of mice that used these "high cycle" device parameters showed reaching behavior by day 5 of training and this subset of mice attained a reaching success rate of $\sim 35 \%$ by day 14 of training.

In experiment 2, we demonstrated the sensitivity of the HASRA to detect lesion-induced impairments. We used the optimized, high cycle parameters in a new group of mice and found that $100 \%$ of mice displayed reaching behavior by day 9 of training. By day 14, mice had once again attained a reaching success rate of $\sim 35 \%$ and allowing additional training to day 22 did not significantly improve this performance $(\sim 37 \%)$. Following stroke, mice continued to spend a significant period of time in the tube but did not attempt to use their hands to reach for the first $2 \mathrm{~d}$ following the surgery. By day 7 poststroke, two of the four mice $(50 \%)$ with stroke were able to show some reaching success, but this was still significantly reduced compared with shams and their own pre-stroke performance ( $\sim 17.5 \%$ success). Overall, this level of reaching success and stroke-induced impairment is comparable to that observed in the single-pellet reaching task (Farr and Whishaw, 2002; Chen et al., 2014).

The HASRA advances previous efforts to automate single-pellet reaching (Torres-Espín et al., 2018) by integrating a robotic pellet dispenser directly within the animals' home-cage environment. This has the benefit of reducing the potential for experimenter-induced stress (Balcombe et al., 2004) and allows the mice to perform the task at any phase of the light-cycle ad libitum. As has been 

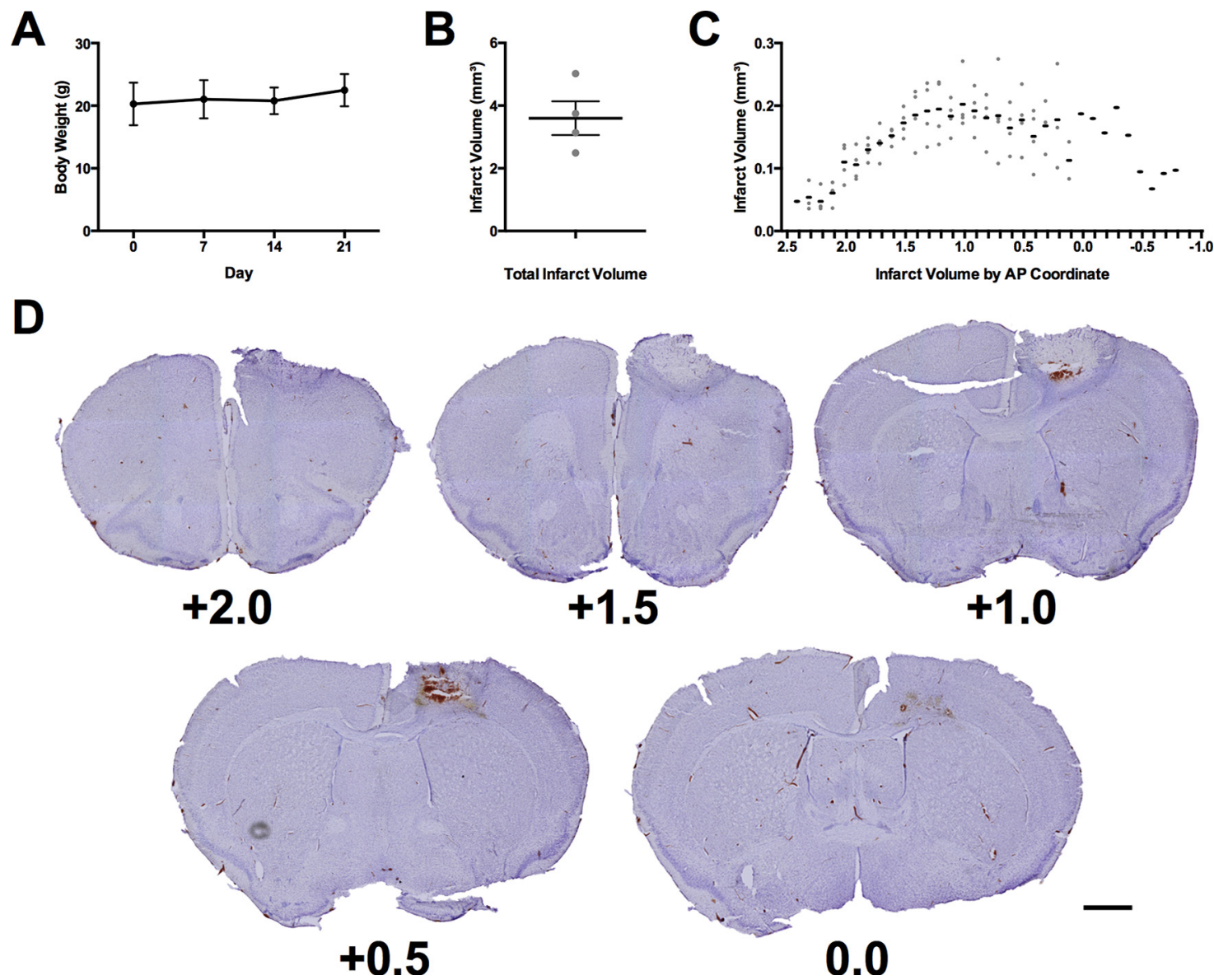

Figure 5. Verification of animal health and lesion induction. $\boldsymbol{A}$, Mean body weight of all mice throughout experiment 2. $\boldsymbol{B}$, Mean total infarct volume of all mice receiving stroke. $\boldsymbol{C}$, Infarct volume of all mice receiving stroke at each anteroposterior coordinate of damage relative to bregma. $\boldsymbol{D}$, Representative cresyl violet images of lesion in the mouse with the total infarct volume closest to the mean $\left(3.75 \mathrm{~mm}^{3}\right)$. Each image is labeled with its distance anterior to bregma in millimeters. Scale bar $=1 \mathrm{~mm}$. The black lines in panels $\boldsymbol{A}-\boldsymbol{C}$ represent the group mean \pm SEM. The gray dots in panels $\boldsymbol{B}, \boldsymbol{C}$ represent the infarct volumes of individual animals. $N=7$ for panel $\boldsymbol{A}$ and $n=4$ in panels $\boldsymbol{B}, \boldsymbol{C}$.

previously observed (Silasi et al., 2018), we found that mice were most inclined to reach during the dark phase, but this is at odds with the majority of behavioral testing paradigms where the experimenter conducts testing during the light phase as a part of their workday. The limited time available to an individual experimenter constrains the amount of training that can be delivered to animals in oneon-one training sessions. By using the HASRA, mice were able to each receive $100+$ min of reaching practice per day, with the total number of mice that could be trained limited only by the number of cages deployed. This high-intensity training was accompanied by individualized training progression for each mouse using a combination of RFID tagging and motorized stages to customize pellet positioning and optimally shape reaching behavior. Each HASRA unit is capable of delivering consistent, yet individualized, training conditions to each animal, potentially reducing the training variability that may be observed with human experimenters (Fouad et al., 2013).

Future development of the HASRA could focus on presenting other types of items, such as traditional reaching pellets, and automating the quantification of mouse performance within the device. Recent developments in markerless tracking methods (Mathis et al., 2018) could enable kinematic analysis of reaching behavior with the use of a high frame-rate camera. Furthermore, convolutional neural nets could be used to automate trial classification (Ryait et al., 2019), which was performed manually in the present study. The current design already employed an image classification scheme to determine when new pellets should be presented during the "single presentation" style in experiment 1. Automated classification of trial outcome would make it feasible to obtain detailed data on every reaching event performed by each mouse, which is beyond the scope of what can be performed by human experimenters given the thousands of reaching events that mice can perform within a single day.

Overall, the HASRA provides novel automation of the classic single-pellet reaching task within a home-cage environment. This task can be used to facilitate motor training experiments on a massive scale that has previously been impossible because of the demands of one-on-one behavioral testing. 


\section{References}

Balbi M, Vanni MP, Silasi G, Sekino Y, Bolanos L, LeDue JM, Murphy TH (2017) Targeted ischemic stroke induction and mesoscopic imaging assessment of blood flow and ischemic depolarization in awake mice. Neurophotonics 4:e035001.

Balcombe JP, Barnard ND, Sandusky C (2004) Laboratory routines cause animal stress. Contemp Top Lab Anim Sci 43:42-51.

Bollu T, Whitehead SC, Prasad N, Walker J, Shyamkumar N, Subramaniam R, Kardon B, Cohen I, Goldberg JH (2019) Automated home cage training of mice in a hold-still center-out reach task. J Neurophysiol 121:500-512.

Brown AR, Teskey GC (2014) Motor cortex is functionally organized as a set of spatially distinct representations for complex movements. J Neurosci 34:13574-13585.

Chen CC, Gilmore A, Zuo Y (2014) Study motor skill learning by single-pellet reaching tasks in mice. J Vis Exp. Advance online publication. Retrieved March 4, 2014. doi: 10.3791/51238.

Clark TA, Sullender C, Jacob D, Zuo Y, Dunn AK, Jones TA (2019) Rehabilitative training interacts with ischemia-instigated spine dynamics to promote a lasting population of new synapses in peri-infarct motor cortex. J Neurosci 39:8471-8483.

Ellens DJ, Gaidica M, Toader A, Peng S, Shue S, John T, Bova A, Leventhal DK (2016) An automated rat single pellet reaching system with high-speed video capture. J Neurosci Methods 271:119127.

Erskine A, Bus T, Herb JT, Schaefer AT (2019) AutonoMouse: high throughput operant conditioning reveals progressive impairment with graded olfactory bulb lesions. PLoS One 14:e0211571.

Farr TD, Whishaw IQ (2002) Quantitative and qualitative impairments in skilled reaching in the mouse (Mus musculus) after a focal motor cortex stroke. Stroke 33:1869-1875.

Fenrich KK, May Z, Hurd C, Boychuk CE, Kowalczewski J, Bennett DJ, Whishaw IQ, Fouad K (2015) Improved single pellet grasping using automated ad libitum full-time training robot. Behav Brain Res 281:137-148.

Fouad K, Hurd C, Magnuson DSK (2013) Functional testing in animal models of spinal cord injury: not as straight forward as one would think. Front Integr Neurosci 7:85.

Gharbawie OA, Gonzalez CLR, Whishaw IQ (2005a) Skilled reaching impairments from the lateral frontal cortex component of middle cerebral artery stroke: a qualitative and quantitative comparison to focal motor cortex lesions in rats. Behav Brain Res 156:125-137.

Gharbawie OA, Gonzalez CLR, Williams PT, Kleim JA, Whishaw IQ (2005b) Middle cerebral artery (MCA) stroke produces dysfunction in adjacent motor cortex as detected by intracortical microstimulation in rats. Neuroscience 130:601-610.

Gu Z, Kalambogias J, Yoshioka S, Han W, Li Z, Kawasawa YI, Pochareddy S, Li Z, Liu F, Xu X, Wijeratne HRS, Ueno M, Blatz E, Salomone J, Kumanogoh A, Rasin MR, Gebelein B, Weirauch MT, Sestan N, Martin JH, et al. (2017) Control of species-dependent cortico-motoneuronal connections underlying manual dexterity. Science 357:400-404.

Guo JZ, Graves AR, Guo WW, Zheng J, Lee A, Rodríguez-González J, Li N, Macklin JJ, Phillips JW, Mensh BD, Branson K, Hantman AW (2015) Cortex commands the performance of skilled movement. Elife 4:e10774.

Hubbard R, Dunthorn J, O'Brien RJ, Tasch D, Tasch U, Zeiler SR (2019) Evaluating skilled prehension in mice using an auto-trainer. J Vis Exp. Advance online publication. Retrieved Sep 12, 2019. doi: $10.3791 / 59784$.

Mathis A, Mamidanna P, Cury KM, Abe T, Murthy VN, Mathis MW, Bethge $M$ (2018) DeepLabCut: markerless pose estimation of user-defined body parts with deep learning. Nat Neurosci 21:1281-1289.
Metz GA, Gonzalez CLR, Piecharka DM, Whishaw IQ (2003) Acute alcohol administration improves skilled reaching success in intact but not 6-OHDA dopamine depleted rats: a subsystems analysis of the motoric and anxiolytic effects of alcohol. Behav Brain Res 142:167-174.

Ryait H, Bermudez-Contreras E, Harvey M, Faraji J, Mirza Agha B, Gomez-Palacio Schjetnan A, Gruber A, Doan J, Mohajerani M, Metz GAS, Whishaw IQ, Luczak A (2019) Data-driven analyses of motor impairments in animal models of neurological disorders. PLoS Biol 17:e3000516.

Sandler M, Howard A, Zhu M, Zhmoginov A, Chen LC (2018) MobileNetV2: inverted residuals and linear bottlenecks. In: 2018 IEEE/CVF conference on computer vision and pattern recognition, pp 4510-4520. Salt Lake City: IEEE.

Silasi G, Hamilton DA, Kolb B (2008) Social instability blocks functional restitution following motor cortex stroke in rats. Behav Brain Res 188:219-226.

Silasi G, Boyd JD, Bolanos F, LeDue JM, Scott SH, Murphy TH (2018) Individualized tracking of self-directed motor learning in group-housed mice performing a skilled lever positioning task in the home cage. J Neurophysiol 119:337-346.

Tennant K, Adkins DL, Donlan N, Asay AL, Thomas N, Kleim J, Jones $T$ (2011) The organization of the forelimb representation of the C57BL/6 mouse motor cortex as defined by intracortical microstimulation and cytoarchitecture. Cereb Cortex 21:865-876.

Torres-Espín A, Forero J, Schmidt EKA, Fouad K, Fenrich KK (2018) A motorized pellet dispenser to deliver high intensity training of the single pellet reaching and grasping task in rats. Behav Brain Res 336:67-76.

Ueno M, Nakamura Y, Li J, Gu Z, Niehaus J, Maezawa M, Crone SA, Goulding M, Baccei ML, Yoshida Y (2018) Corticospinal circuits from the sensory and motor cortices differentially regulate skilled movements through distinct spinal interneurons. Cell Rep 23:1286-1300.e7.

Whishaw IQ (1992) Lateralization and reaching skill related: results and implications from a large sample of Long-Evans rats. Behav Brain Res 52:45-48.

Whishaw IQ (2000) Loss of the innate cortical engram for action patterns used in skilled reaching and the development of behavioral compensation following motor cortex lesions in the rat. Neuropharmacology 39:788-805.

Whishaw IQ, Pellis SM (1990) The structure of skilled forelimb reaching in the rat: a proximally driven movement with a single distal rotatory component. Behav Brain Res 41:49-59.

Whishaw IQ, Whishaw P, Gorny B (2008) The structure of skilled forelimb reaching in the rat: a movement rating scale. J Vis Exp. Advance online publication. Retrieved Aug 8, 2008. doi: 10.3791/ 816.

Whishaw IQ, Faraji J, Kuntz J, Mirza Agha B, Patel M, Metz GAS, Mohajerani MH (2017) Organization of the reach and grasp in head-fixed vs freely-moving mice provides support for multiple motor channel theory of neocortical organization. Exp brain Res 235:1919-1932.

Wong CC, Ramanathan DS, Gulati T, Won SJ, Ganguly K (2015) An automated behavioral box to assess forelimb function in rats. $J$ Neurosci Methods 246:30-37.

Woodard CL, Bolaños F, Boyd JD, Silasi G, Murphy TH, Raymond LA (2017) An automated home-cage system to assess learning and performance of a skilled motor task in a mouse model of Huntington's disease. eNeuro 4:ENEURO.0141-17.2017.

Xu T, Yu X, Perlik AJ, Tobin WF, Zweig JA, Tennant K, Jones T, Zuo $Y$ (2009) Rapid formation and selective stabilization of synapses for enduring motor memories. Nature 462:915-919. 